\title{
Aurélien Ruellet, La Maison de Salomon. Histoire du patronage scientifique et technique en France et en Angleterre au XVII siècle
}

Rennes, Presses universitaires de Rennes, 2016, 340 p.

\section{Brice Cossart}

\section{OpenEdition}

Journals

Édition électronique

URL : http://journals.openedition.org/artefact/3721

DOI : 10.4000/artefact.3721

ISSN : 2606-9245

Éditeur :

Association Artefact. Techniques histoire et sciences humaines, Presses universitaires du Midi

Édition imprimée

Date de publication : 15 mars 2019

Pagination : 343-347

ISBN : 978-2-8107-0623-5

ISSN : $2273-0753$

\section{Référence électronique}

Brice Cossart, «Aurélien Ruellet, La Maison de Salomon. Histoire du patronage scientifique et technique en France et en Angleterre au xvı" siècle », Artefact [En ligne], 9 | 2018, mis en ligne le 04 mars 2020, consulté le 29 novembre 2020. URL : http://journals.openedition.org/artefact/3721 ; DOI : https:// doi.org/10.4000/artefact.3721

Ce document a été généré automatiquement le 29 novembre 2020.

\section{cc) (†)}

Artefact, Techniques, histoire et sciences humaines est mise à disposition selon les termes de la Licence Creative Commons Attribution - Pas d'Utilisation Commerciale - Pas de Modification 4.0 International. 


\section{Aurélien Ruellet, La Maison de Salomon. Histoire du patronage scientifique et technique en France et en Angleterre au XVII siècle}

Rennes, Presses universitaires de Rennes, 2016, 340 p.

\section{Brice Cossart}

1 La Maison de Salomon est la publication d'une thèse soutenue en 2014 par Aurélien Ruellet au Centre d'études supérieures sur la Renaissance de Tours, sous la direction de Pascal Brioist, dont le titre reprend une image au cœur du récit utopique de Francis Bacon dans sa Nouvelle Atlantide (1627). Offrant un soutien plus ou moins institutionnalisé à une multitude d'acteurs dédiés à l'avancement des sciences, cette organisation imaginée par le fameux homme d'État anglais a souvent été considérée par l'historiographie comme une vision anticipant les futures académies des sciences. Or, Aurélien Ruellet nous démontre que, bien que fictive, la maison de Salomon s'inspirait d'une certaine réalité de l'époque qu'il propose de mettre en lumière à travers une étude portant sur les multiples formes qu'a pu revêtir le patronage scientifique et technique en France et en Angleterre avant la création de la Royal Society (1660) et de l'Académie royale des sciences (1666). Il en résulte un travail original et intéressant à plusieurs titres.

D'abord, ce livre parvient à redéfinir les contours de ce que fut le patronage scientifique et technique dans la première moitié $d u x$ III $^{e}$ siècle en révélant les multiples ressources utilisées par les praticiens des sciences et des techniques pour soutenir leur activité. Le mécénat aristocratique, d'ordinaire omniprésent en histoire des sciences, ne s'avère finalement jouer qu'un rôle secondaire dans les dizaines de parcours de savants analysés par Aurélien Ruellet. Il ressort de son étude que de nombreuses carrières s'appuyèrent sur une pluralité de protections imbriquées de manière plus ou moins complexe selon les cas : mécénat monarchique avec position officielle dans l'appareil d'État, patronage coutumier permettant de favoriser les 
démarches administratives des savants, ou encore privilèges d'invention protégeant une entreprise capitaliste. Grâce à cette définition élargie du patronage scientifique et technique, la Maison de Salomon est en mesure de mieux saisir l'environnement social entourant la transformation des pratiques savantes à cette époque charnière qui précéda la mise en place des célèbres académies scientifiques. Le cadre spatio-temporel est astucieusement choisi puisqu'il porte sur une période souvent délaissée par l'historiographie. En outre, la comparaison entre la France et l'Angleterre se montre tout à fait pertinente dans la mesure où elle révèle de nombreux entremêlements d'individus, d'idées et de modèles de patronage entre des États voisins, tous deux très dynamiques en matière de production savante. L'auteur choisit de restreindre son périmètre d'étude aux sciences et techniques relevant de savoirs mathématiques tels que les identifie le projet baconien de maitrise du monde naturel. Il parvient ainsi à pré server la cohérence d'une culture savante aux multiples facettes, réunissant astronomes, ingénieurs, horlogers ou encore fabricants d'arquebuses. Il s'inscrit de la sorte dans ce mouvement récent de reconsidération positive de la contribution des artisans et autres praticiens des mathématiques à l'activité intellectuelle de l'époque moderne ${ }^{1}$.

3 La première des trois grandes parties de l'ouvrage est consacrée à l'analyse du patronage aristocratique. Plusieurs formes de protection sont identifiées dans ce registre : la domesticité, souvent à travers une position de tuteur ou de chapelain, plaçait le savant dans une position de dépendance et d'infériorité que tous n'étaient pas prêts à accepter, tandis que la commensalité et l'hospitalité, symboliquement plus égalitaires, constituèrent les socles, souvent peu visibles, de nombreuses carrières. L'un des enjeux les plus cruciaux du patronage aristocratique se situa au niveau de la promotion d'une sociabilité savante, notamment à travers l'émergence des cénacles parisiens à partir des années 1630. Certains aristocrates, tel le maitre des requêtes Henri-Louis Habert de Montmor, accueillirent régulièrement savants et beaux-esprits de la République des Lettres afin de débattre ou de réaliser des expériences. Cependant, l'auteur note la frustration des savants à dépendre d'une aristocratie plus portée sur le spectacle que sur l'avancement des connaissances. Finalement, la présence en France de nombreux exilés anglais lors de l'Interrègne conduisit à la critique du modèle de sociabilité savante des cénacles parisiens et favorisa l'émergence, sous l'autorité de 1 'aristocrate Robert Boyle, d'un nouveau modèle fondé sur l'établissement de faits expérimentaux, plus tard institutionnalisé par la création de la Royal Society. En d'autres termes, l'auteur explique que la mise en place des académies par les monarchies anglaise et française répondait, du point de vue des savants, aux défauts et aux limites du patronage aristocratique. Ce dernier est ensuite analysé jusque dans ses liens avec la culture de l'imprimé, à travers la pratique de la dédicace. Loin d'être systématiquement des témoignages d'une recherche de mécénat, les dédicaces y apparaissent comme des textes codifiés aux intentions multiples parmi lesquelles figurent l'espoir d'obtention d'une charge publique, le signalement public d'allégeance ou d'amitié, ou encore la promotion commerciale du livre. Souvent, le dédicataire était ciblé en tant que détenteur d'une charge publique plus qu'en tant que noble, ce qui démontre combien les patronages aristocratique et administratif étaient entremêlés.

4 La seconde partie de l'ouvrage s'interroge sur le rôle de l'État dans les dynamiques de patronage scientifique et technique. Or, dans cette période qui précède la création des académies, l'appareil monarchique apparaît déjà comme un acteur incontournable du marché de l'emploi des savants et des praticiens des mathématiques. Ce patronage que 
l'auteur nomme "administratif » offrait un accès aux abondantes ressources de l'État, mais il s'inscrivait avant tout dans une quête de savoirs orientés vers l'applicatif, ce qui confirme la pertinence du choix de placer l'étude à la confluence des sciences et des techniques. La Monarchie s'associait volontiers au savant à condition que ce dernier serve la puissance de l'État, notamment dans l'armée ou la marine. L'ingénierie civile, ainsi que les eaux et fontaines, par leurs dimensions spectaculaire et utilitaire, s'inscrivaient dans cette même perspective du renforcement du pouvoir royal par les sciences mathématiques. En revanche, les charges d'enseignement sous contrôle monarchique demeuraient peu nombreuses, circonscrites à la cour ainsi que, dans le cas français, au Collège royal. Parmi ces savants et techniciens au service de l'État, l'auteur identifie de nombreux ingénieurs, mais aussi une pléthore d'artisans spécialisés: horlogers, orfèvres, artilleurs, fabricants d'armes ou d'instruments mathématiques, certains de ces individus servaient dans les structures étatiques tandis que d'autres obtinrent le privilège de s'installer à la cour anglaise ou dans la galerie du Louvre. Enfin, un cas d'étude vient illustrer comment s'imbriquaient les logiques de patronage privées et étatiques. La querelle des longitudes déclenchées en 1634 par le médecin, astronome et mathématicien Jean-Baptiste Morin permet de saisir les enjeux autour de l'accès aux ressources du patronage aristocratique et administratif. L'administration royale, incapable de juger les propositions de Morin sur un sujet pourtant si capital à ses ambitions navales, se trouva contrainte de déléguer l'expertise à un comité de savants et de courtisans. De ce fait, les oppositions et les luttes internes au mécénat aristocratique débordaient dans le champ administratif.

5 La troisième et dernière partie, la plus novatrice de l'ouvrage, s'intéresse aux privilèges d'invention octroyés par les monarchies anglaise et française aux entreprises techniques. D'abord, l'auteur parvient à convaincre de la légitimité à traiter de cette thématique dans la continuité des deux précédentes. En effet, on y retrouve d'abord un certain nombre d'acteurs communs, tant du côté des inventeurs que de celui des courtisans et des hommes d'État. En outre, le privilège d'invention s'avère bien être une forme de protection, en l'occurrence juridique, allouée aux savants et techniciens afin de favoriser leur activité par le biais du marché. Mais surtout, il met en jeu d'importants mécanismes de patronage car, s'il existait bien des procédures d'examen des requêtes, l'octroi des privilèges d'invention s'effectuait principalement sur la base de critères institutionnels et juridiques. Comme le cas de la querelle des longitudes l'a mis en évidence, l'État ne disposait pas d'un personnel capable d'évaluer de manière approfondie les aspects techniques de l'innovation et, par conséquent, la logique qui prévalait était celle de la faveur, avec tous les rapports de patronage qu'elle impliquait. L'étude souligne le rôle essentiel de certains courtisans qui, grâce à leur accès privilégié au roi, constituaient des intermédiaires extrêmement précieux pour les inventeurs. Ce furent en particulier les secrétaires d'État en France, les maîtres des requêtes en Angleterre, ainsi que les gentilshommes de la Chambre du Roi. Ce fonctionnement particulier donna forme à des associations entrepreneuriales entre techniciens, capitalistes et courtisans. Pour l'illustrer, l'ouvrage suit les parcours de deux inventeurs à répétition, David Ramsey et Pierre de Beringhen, tous deux techniciens, alchimistes et hommes à projet disposant d'une certaine proximité avec leur souverain.

6 Cette monographie constitue donc une étude à la fois originale, puisqu'elle s'appuie sur de nombreuses sources primaires, et complète, puisqu'elle envisage le patronage scientifique et technique sous une multitude d'angles complémentaires. On déplorera seulement le manque de lisibilité de certains graphes représentant des réseaux de 
savants et de courtisans. On regrettera peut-être également l'absence d'une mise en perspective du sujet avec la période antérieure. En effet, les principaux éléments - les patronages aristocratique et étatique, les privilèges d'invention - constituaient déjà au $\mathrm{XVI}^{\mathrm{e}}$ siècle des piliers de l'activité scientifique et technique. D'ailleurs, pour mieux symboliser ce poids de l'héritage du siècle précédent, il faut rappeler que les écrits de Francis Bacon, qui introduisent si bien le présent ouvrage, furent inspirés de sources antérieures, aussi bien anglaises ${ }^{2}$ qu'espagnoles $^{3}$. Dès lors se pose la question de la spécificité de la période étudiée qui ne semble pas véritablement en rupture avec la fin $\mathrm{du} \mathrm{XVI}^{\mathrm{e}}$ siècle. Néanmoins, cette remarque répond plutôt à un souhait d'exploration suscité par la lecture stimulante de ce livre qu'à une véritable lacune. Les recherches d'Aurélien Ruellet embrassent un ensemble de sources très vaste auquel il était pertinent de donner une borne chronologique initiale.

Il va sans dire que cet ouvrage contribue de manière significative à l'historiographie des sciences et des techniques ainsi qu'à celle de la construction de l'État. La Maison de Salomon présente une vue transversale unique sur les multiples facettes du patronage des sciences et des techniques au sein de la société de cour du XVII siècle, dont les mécanismes de promotion sociale sont analysés avec finesse. La pertinence de l'objet $\mathrm{d}$ 'étude situé au carrefour entre les sciences et les techniques touchant aux savoirs mathématiques renforce cette vieille hypothèse de Zilsel, récemment sortie de l'ombre, quant à la participation des milieux techniciens aux transformations des pratiques scientifiques de la première modernitét. Même si le rôle prépondérant de l'État dans les dynamiques de patronage n'est pas surprenant, cette étude a l'important mérite d'en fixer le cadre de fonctionnement. Enfin, elle attire l'attention sur un acteur un peu moins attendu du développement scientifique et technique : il s'agit du marché, abordé à plusieurs reprises notamment à travers les enjeux autour de la promotion des livres imprimés, de l'exploitation commerciale des privilèges d'invention, et plus discrètement à travers le droit des artisans de la Galerie du Louvre à poursuivre un commerce privé en sus des financements monarchiques. Enfin, le signe indubitable de l'intérêt de cet ouvrage est le sentiment que sa lecture suscite en faveur d'un approfondissement des recherches sur cette thématique du patronage et des divers moyens de promotion de l'activité scientifique et technique à l'époque moderne.

\section{NOTES}

1. Pamela H. sмiтh, The Body of the Artisan: Art and Experience in the Scientific Revolution, Chicago, University of Chicago Press, 2004 ; Pamela O LONG, Artisan/Practitioners and the Rise of the New Sciences, 1400-1600, Corvallis, Oregon State University Press, 2011.

2. Deborah E. HARKNESS, The Jewel House. Elizabethan London and the Scientific Revolution, New Haven ; London, Yale University Press, 2007.

3. Juan PIMENTEL, "The Iberian Vision: Science and Empire in the Framework of a Universal Monarchy, 1500-1800», Osiris 2nd series, $n^{\circ} 15$, Nature and Empire: Science and the Colonial Enterprise, 2000, p. 17-30. 
4. Edgar ZILSEL, "The Genesis of the Concept of Scientific Progress ", Journal of the History of Ideas, vol. 6, $\mathrm{n}^{\circ}$ 3, 1945, p. 325-349; Pamela O LoNG, Artisan/Practitioners and the Rise of the New Sciences, 1400-1600, Corvallis, Oregon State University Press, 2011. 